\title{
Quantum confinement effects on the ordering of the lowest-lying excited states in conjugated chains
}

\section{Z. Shuai and J.L. Brédas}

Service de Chimie des Matariaux Nouveaux

Centre de Recherche en Electronique et Photonique Moléculaires

Universitat de Mons-Hainaut, Place du Parc 20, B-7000 Mons, Belgium

S. K. Pati and S. Ramasesha

Solid State and Structural Chemistry Unit

Indian Institute of Science, Bangalore 560012, India

\begin{abstract}
The symmetrized density matrix renormalization group approach is applied within the extended Hubbard-Peierls model (with parameters $U / t, V / t$, and bond alternation $\delta$ ) to study the ordering of the lowest one-photon $\left(1^{1} B_{u}^{-}\right)$and two-photon $\left(2^{1} A_{g}^{+}\right)$states in one- dimensional conjugated systems with chain lengths, $N$, up to $N=80$ sites. Three different types of crossovers are studied, as a function of $U / t$, $\delta$, and $N$. The $U$-crossover emphasizes the larger ionic character of the $2 A_{g}$ state compared to the lowest triplet excitation. The $\delta$ crossover shows strong dependence on both $N$ and $U / t$. The $N$-crossover illustrates the more localized nature of the $2 A_{g}$ excitation relative to the $1 B_{u}$ excitation at intermediate correlation strengths.
\end{abstract}

PACS Numbers: 71.10.-w, 71.24.+q, 78.66.Qn

Recently, much attention has focused on the luminescence properties of conjugated organic materials because of their potential for application in display devices [1]. These studies have underscored the importance of the structure of low-lying electronic excited states. Specifically, a major parameter is the relative ordering of the lowest dipole allowed singlet $\left(1^{1} B_{u}^{-}\right)$state and the lowest dipole forbidden singlet $\left(2^{1} A_{g}^{+}\right)$state, in the light of Kasha's rule which relates molecular fluorescence to the lowest excited singlet state.

It is well established that correlated electron systems behave differently from independent electron systems, especially in the case of excitations. Earlier work has shown that the lowest optically forbidden excited state $2 A_{g}$ lies below the optically allowed excited state $1 B_{u}$ in polyene molecules [2] ( thus preventing any significant luminescence in such compounds ), while an independent electron model gives the opposite picture; similar results have been found by Periasamy et al. in the case of polycrystalline sexithienyl [3] or Lawrence et al. in single crystal polydiacetylene [4]. These examples serve as evident 
manifestation of electron correlation in conjugated molecules. The influence of electron correlation has also been considered as the main origin of lattice dimerization leading to the view that conjugated polymers are rather Mott insulators than Peierls insulators [5]. In the context of third-order nonlinear optical response and photo-induced absorption, the role of higher-lying excited states derived from correlated electron models has also been emphasized [6].

It is also important to stress that the electronic and optical properties of conjugated oligomers and polymers differ depending on whether the compounds are in the gas phase, in solution, or in the solid state [7]. The chemical environment affects the geometric structure as well as the electron correlation strength, the latter via dielectric screening [8]. Furthermore, the characteristics of the conjugation defects present in oligomers depend on chain length, which emphasizes the influence of quantum size effects [9].

In view of these features, we believe that these three factors: (i) geometric structure, (ii) strength of electron correlation, and (iii) quantum confinement, are most relevant for the study of the photo- and electro-luminescence response in organic conjugated chains.

Previous studies of the $1 B / 2 A$ crossover behavior have been carried out for short chain systems, so that in the independent electron limit, the $2 A$ energy is significantly higher than that of $1 B$ due to the discreteness of the molecular orbital energy spectrum. In this zero $U$ limit, the $2 A$ state corresponds to single $H O M O$ to $L U M O-1$ (or $H O M O+1$ to $L U M O$ ) excitation while $1 B$ is a $H O M O$ to $L U M O$ excitation. According to previous results [10], as electron correlation $U$ is turned on, the gap between the ground state and the $2 A$ state narrows while the gap to the $1 B$ state increases; the states thus cross at a given Hubbard correlation strength $U_{c}$. This, we refer to as the $U$-crossover. However, for an infinite chain, the $2 A$ and $1 B$ states both occur at the same energy in the H'uckel limit $(U=0)$. If the $2 A$ and $1 B$ states were evolving in a manner identical to that in the short chains, these states would never cross with increasing $U$. Thus, for a given $U$, their must occur a crossover from the short chain behavior to the long chain behavior; this we refer to as the $N$-crossover.

It was noted by Soos, Ramasesha and Galv'ao [11] from exact diagonalization studies of short chains that a similar crossover occurred with variation of the bond-alternation parameter $\delta$, which we refer to as the $\delta$-crossover. The $\delta$-crossover was studied by monitoring the optical gap and the lowest singlet-triplet ( spin ) gap; the critical $\delta_{c}$ for a given 
correlation strength was determined by the value of $\delta$ at which the optical gap equals twice the spin gap. These authors further described the system as behaving band-like for $\delta$ values above $\delta_{c}$ and correlated-like for $\delta$ values below $\delta_{c}$. However, as was pointed out in Ref. [12], increasing bond alternation does not lead to the band picture, because the binding energy of the $1 B$ exciton increases with increasing $\delta$, an obvious indication that electron correlation increases at the same time.

In this Letter, we present a thorough study that encompasses the three kinds of crossovers, namely the $U, N$, and $\delta$ crossovers in conjugated chains, by employing the symmetrized density matrix renormalization group ( SDMRG) theory. The SDMRG approach [13] is currently the most reliable many-body method for calculating the low-lying excited states with high accuracy for relatively large systems and for a wide range of model parameters. The model Hamiltonian in this study is the extended Hubbard-Peierls Hamiltonian which reads:

$$
\hat{H}=\sum_{<i j>, \sigma} \eta_{i}\left[-t\left(\hat{a}_{i, \sigma}^{\dagger} \hat{a}_{j, \sigma}+\hat{a}_{j, \sigma}^{\dagger} \hat{a}_{i, \sigma}\right)\right]+\sum_{i} \frac{1}{2} U \hat{n}_{i}\left(\hat{n}_{i}-1\right)+\sum_{<i j>} \eta_{i}\left[V\left(\hat{n}_{i}-1\right)\left(\hat{n}_{j}-1\right)\right.
$$

where $\eta_{i}=\left[1-\delta(-1)^{i}\right], \delta$ is the dimensionless dimerization parameter, $U$ is the onsite Hubbard repulsion ( in units of $t$, the nearest-neighbor hopping integral ) and $V$ is the nearest-neighbor charge density-charge density interaction. The $\delta$ term serves as a structural parameter in the simplest way, if we assume linear electron-lattice coupling in the static limit; as has been pointed out before, the $V$-term is crucial to the understanding of the optical excitation spectrum, namely the excitonic effect [14]. The present model can be regarded as the minimal correlated model for conjugated systems. Note that, the meaningful phase corresponds to the $B O W$ ( bond-order wave ) regime, namely, $V<U / 2$ [15]. By comparing experimental data for a series of polyene molecules, we find that the parameter set $(t, U, V, \delta)=(2.4 \mathrm{eV}, 7.2 \mathrm{eV}=3 t, 0.4 U, 0.07)$ gives the best fit for the $1 B$, $2 A$, and even higher energy $A(m A)$ states [16]. We thus set $V / U=0.4$ without losing generality.

The density matrix renormalization group method is the most accurate numerical method for determining the ground and low-lying excited states of quasi-one-dimensional correlated electron systems with short range interactions [17]. In the usual DMRG procedure, it is difficult to target the $1 B_{u}$ state as there are many states that appear between it and the ground state, with the number of these states increasing with $U$ and chain length $N$. However, in a symmetrized DMRG technique that exploits spin parity, $C_{2}$ 
symmetry, and electron-hole symmetry, the $1 B_{u}$ state is the lowest state in the subspace ${ }^{e} B_{u}^{-}$. Incorporating these three symmetries thus allows us to determine the $1 B_{u}^{-}$and the $2 A_{g}^{+}$state energies with unprecedented accuracy for chains of up to 80 sites. We choose to truncate the space of density matrix eigenstates to $100(m=100)$ in most cases. For smaller $U$ and $\delta$, however, we choose a larger value of $m(=150)$ in order to achieve consistent accuracy.

We contrast the $U$-crossover for short $(N=8)$ and long $(N=80)$ chains for fixed alternation $\delta=0.07$ in Fig. 1. It is well known that in the strong correlation limit, the $2 A$ state becomes a spin excitation which is gapless in the limit $\delta=0$ and this state can be described as composed of two triplets. Thus, increase in correlation strength should show a decrease in the $2 A$ energy [18]. However, we note that in the $N=8$ chain, the two-photon state energy remains nearly constant before decreasing for values of $U / t$ larger than 2.0. In the longer chain, the $2 A_{g}$ energy increases even more rapidly with increasing correlation strength, than the $1 B_{u}$ energy. This implies a substantial ionic contribution to the $2 A_{g}$ state in long chains besides the covalent triplet-triplet contribution. This result constitutes the first clear illustration of the importance of quantum size effects. We find, however, that the critical correlation strength, $U_{c}$, at which the crossover occurs is nearly independent of the chain length $N$; in both $N=8$ and $N=80$ cases, $U_{c}$ is around 2.5t.

For fixed correlation strength $(U / t=3$ and 4 ), we present the $\delta$-crossover results for $N=8$ and 80 in Fig. 2. We find that the critical $\delta$ value, $\delta_{c}$, strongly depends on chain length. For $U / t=3$, the $\delta_{c}$ values are found to be 0.15 and 0.09 for $N=8$ and 80 , respectively; for $U / t=4$, they are 0.32 and 0.22 . Thus, $\delta_{c}$ has both strong $N$ and $U$ dependence. We also show in Fig. 2 the crossover behavior between the $1 B_{u}$ energy and twice the lowest triplet energy, $E_{T}$. This crossover occurs at systematically smaller $\delta$ values, again emphasizing the larger ionic character present in the $2 A_{g}$ state compared to the lowest triplet state.

Most interestingly, we find one more crossover behavior, which is the $N$-crossover, in the case of intermediate $U / t$ and medium to large $\delta$ values. We observe that the $1 B_{u}$ and $2 A_{g}$ states cross over for fixed $U / t$ and $\delta$ as a function of $N$, the chain length. The critical lengths are actually fairly insensitive to $U$ and $\delta$. In Figs. 3a $(U / t=3, \delta=0.12)$ and $3 \mathrm{~b}$ ( $U / t=4.0, \delta=0.27$ ), we find this crossover for $N=14$ and $N=12$, respectively. This is a direct theoretical observation of quantum confinement induced crossover. It is related 
to the fact that the $2 A_{g}$ excitation is more local in character with a shorter characteristic length than the $1 B_{u}$ state. Thus, the $1 B_{u}$ excitation is stabilized over longer length scales than the $2 A_{g}$ excitation. This is seen as a more rapid saturation in the $2 A_{g}$ energy compared to the $1 B_{u}$ energy, as a function of chain length. We note that this crossover can also be seen from Fig. 2 where the $\delta_{c}$ values show a decrease in going from $N=8$ to $N=80$. This behavior can only exist for intermediate correlation strength: for weak correlation, there does not exist any crossover and $2 A_{g}$ lies above the $1 B_{u}$ state for all chain lengths as seen from Fig.1; at large values of $U / t$, we are in the atomic limit, a crossover is not expected and the quantum size effects are largely suppressed. It has been widely accepted that the conjugated molecules fall in the intermediate correlation regime; thus, the confinement-induced crossover is realistic.

It is most relevant in this context to stress that, for smaller $\delta$ values, even though no crossover occurs, the $1 B / 2 A$ gap decreases in long chains. When considering the parameters adapted to polyenes $(U / t=3, V=0.4 U, \delta=0.07)$, both $1 B_{u}$ and $2 A_{g}$ state energies decrease with $N$, but $2 A_{g}$ state saturates in a way much faster than $1 B_{u}$, because the former ( mostly covalent ) is more localized than the latter. Consequently, it would be inappropriate to extrapolate the $1 B / 2 A$ gap in polyacetylene from data obtained on short chains [19]; our results imply that the actual gap is significantly smaller than the result of such an extrapolation.

To conclude, we have employed the accurate numerical density matrix renormalization group technique with symmetry adaptation to study the ordering of the lowest onephoton and two-photon states in conjugated oligomers and polymers within an extended Hubbard-Peierls model. Three kinds of crossover, namely a $U$-crossover, a $\delta$-crossover, and a $N$-crossover, have been demonstrated. The $N$-crossover is related to quantum finite size effects and crucially depends on the characteristic length of the excitations; this characteristic length is of the same order as the chain length at intermediate correlation strengths and degrees of dimerization.

\section{Acknowledgments}

This work is partly supported by the Belgian Prime Minister Services for Scientific, Technical, and Cultural Affairs (Interuniversity Attraction Pole 4/11 in Supramolecular Chemistry and Catalysis), FNRS/FRFC (Loterie Nationale), and an IBM Academic Joint Study. The work in Bangalore is partly supported by the Jawaharlal Nehru Center for 
Advanced Scientific Research. 


\section{References}

1. J. H. Burroughes, D. D. C. Bradley, A. R. Brown, R. N. Marks, K. Mackay, R. H. Friend, P..L. Burn, and A. B. Holmes, Nature 347, 539 (1990); G. Gustafsson, Y. Cao, G. M. Treacy, F. Klavetter, N. Colaneri, and A. J. Heeger, Nature 357, 477 (1992).

2. B. S. Hudson and B. E. Kohler, Chem. Phys. Lett. 14, 229 (1972); J. Chem. Phys. 59, 4984 (1973).

3. N. Periasamy, R. Danieli, G. Ruani, R. Zamboni, and C. Taliani, Phys. Rev. Lett. 68, 919 (1992).

4. B. Lawrence, W. E. Torruellas, M. Cha, M. L. Sundheimer, G. I. Stegeman, J. Meth, S. Etemad, and G. Baker, Phys. Rev. Lett. 73, 597 (1994).

5. S. Mazumdar and D. K. Campbell, Phys. Rev. Lett. 55, 2067 (1985); C. Q. Wu, X. Sun, and K. Nasu, Phys. Rev. Lett. 63, 2534 (1989).

6. J. M. Leng, S. Jeglinski, X. Wei, R. E. Benner, Z. V. Vardeny, F. Guo, and S. Mazumdar, Phys. Rev. Lett. 72, 156 (1994); F. Guo, M. Chandross, and S. Mazumdar, Phys. Rev. Lett. 74, 2096 (1995); S. N. Dixit, D. Guo, and S. Mazumdar, Phys. Rev. B 43, 6781 (1991).

7. O. Dippel, V. Brandl, H. Bssler, R. Danieli, R. Zamboni, and C. Taliani, Chem. Phys. Lett. 216, 418 (1993).

8. S. R. Marder, C. B. Gorman, F. Meyers, J. W. Perry, G. Bourhill, J. L. Brédas, and B. M. Pierce, Science 265, 632 (1994).

9. J. L. Brédas and A. J. Heeger, Chem. Phys. Lett. 154, 56 (1989).

10. a) G. W. Hayden and E. J. Mele, Phys. Rev. B 34, 5484 (1986); b) D. Baeriswyl, D. K. Campbell, and S. Mazumdar, in Conjugated Conducting Polymers, edited by H. Kiess, Springer-Verlag (Berlin, 1992), and references therein.

11. Z. G. Soos, S. Ramasesha, and D. S. Galväo, Phys. Rev. Lett. 71, 1609 (1993). 
12. Z. Shuai, S. K. Pati, W. P. Su, J. L. Brédas, and S. Ramasesha, Phys. Rev. B. (1997).

13. S. Ramasesha, S. K. Pati, H. R. Krishnamurthy, Z. Shuai, and J. L. Brédas, Phys. Rev. B, 54, 7598 (1996).

14. D. Guo, et al., Phys. Rev. B 48, 1433 (1993).

15. S. Mazumdar and D. K. Campbell, Phys. Rev. Lett. 55, 2067 (1985); see also Ref. $[10 \mathrm{~b}]$.

16. Z. Shuai, A. B. Saxena, J. L. Brédas, A. R. Bishop, and B. E. Kohler, to be published.

17. S. R. White, Phys. Rev. Lett. 69, 2863 (1992); Phys. Rev. B 48, 10345 (1993).

18. D. Mukhopadhyay, G. W. Hayden, and Z. G. Soos, Phys. Rev. B 51, 9476 (1995).

19. B. E. Kohler, J. Chem. Phys. 93, 5838 (1990). 


\section{Figure Captions}

Figure 1. Crossover on $U$ for $\delta=0.07$.

Figure 2. Crossover on $\delta$ for (a) $U / t=3$; (b) $U / t=4$.

Figure 3. Crossover on $N$ for (a) $U / t=3$ and $\delta=0.12$; (b) $U / t=4$ and $\delta=0.27$. 